\title{
Ángulo de Inclinación del Fémur en el Hombre y su Relación con la Coxa Vara y la Coxa Valga
}

\author{
Angle of Inclination of Femur in Man and its Relationship with Coxa Vara and Coxa Valga
}

"Luiz Carlos Buarque de Gusmão; * Célio Fernando de Sousa Rodrigues; ${ }^{* * * *}$ Josefa Sileda Martins \& ${ }^{* * * * *}$ Anselmo José da Silva

GUSMÃO, L. C. B.; SOUSA-RODRIGUES, C. F.; MARTINS, J. S. \& SILVA, A. J. Ángulo de inclinación del fémur en el hombre y su relación con la coxa vara y la coxa valga. Int. J. Morphol., 29(2):389-392, 2011.

RESUMEN: Los autores realizan mediciones en fémures aislados, realizando comparación de los datos obtenidos con los resultados encontrados en la literatura investigada, con el objeto de determinar de forma porcentual los valores limítrofes para clasificar la coxa como vara o valga. Fueron analizados 56 fémures de cadáveres adultos, siendo determinado el ángulo de inclinación del fémur, que fue medido a través de un Goniómetro. Utilizamos el patrón poblacional normal de $66 \%$ (o test Z) para determinar cuál es el intervalo donde se encuentra el alineamiento ideal. En las mediciones de los ángulos de inclinación de los 56 fémures, los valores se encontraban entre 117,67 grados y 136,67 grados. Utilizando el patrón poblacional normal de 66\% (o test Z), verificamos que el intervalo ideal (o normal) se encuentra entre 117 grados y 136 grados, siendo éste el intervalo donde se encuentra el alineamiento ideal. En este intervalo encontramos 47 fémures (84\%). La determinación normal del ángulo entre 117 grados y 136 grados, posibilita clasificar de coxa valga cuando el ángulo de inclinación está debajo de 177 grados y coxa vara encima de 136 grados.

PALABRAS CLAVE: Genu valgo; Genu varo; Osteotomías del fémur; Anatomía.

\section{INTRODUCCIÓN}

La posición de coxa valga y de coxa vara depende de una serie de factores genéticos y anatómicos de los miembros inferiores, llevando a las deformidades de las rodillas, conocidas como genu valgo y genu varo respectivamente. En el caso de la coxa vara, podemos verificar una variedad de combinaciones que resultan en esta situación de varismo, que según Kendal et al. (1995) son la rotación medial del fémur y pronación de los pies. En el caso de la coxa valga, son similares siendo que en sentido contrario a las de la coxa vara, que son la rotación lateral de fémures y supinación de los pies.

El ángulo de inclinación del fémur consiste en un ángulo determinado entre el eje del cuello del fémur con el eje longitudinal de la diáfisis femoral, que según Hamil \& Knutzen (1999), se ubica entre $90^{\circ}$ y $135^{\circ}$. Para Kapandji (2001), una inclinación mayor de $125^{\circ}$ se denomina coxa valga, y una inclinación menor de $120^{\circ}$ se llama coxa vara.
Rocha (1995) cita que coxa valga tiene como la principal característica el pie en eversión y en la coxa vara el pie en inversión. Entre los factores más significativos que contribuyen al aparecimiento de las alteraciones citadas, está, según Field et al. (2000) y Karpandji, la disminución o aumento del ángulo de inclinación de la cabeza del fémur en relación a su cuerpo.

Lapìerre (1982) relata el ángulo de inclinación midiendo $128^{\circ} \mathrm{y}$, con la disminución del ángulo debajo de los $90^{\circ}$, se forma la coxa vara, y para la formación de la coxa valga este ángulo debe estar situado entre $150^{\circ}$ y $160^{\circ}$.

Para Kendal et al., el problema del aparecimiento de desvíos al nivel de los muslos y, consecuentemente, de las rodillas, estaría en alteraciones al nivel de los cuadriles y no en el ángulo de inclinación del fémur, ya que, para que no ocurran desvíos, los mismos deben conservar una postura

* Máster y Doctor en Anatomía Humana. Profesor de la Universidad Federal de Alagoas y del Instituto Batista de Enseñanza Superior de Alagoas; Miembro Titular del Colegio Brasileño de Cirujanos, Brasil.

** Máster y Doctor en Anatomía Humana; Profesor de la Universidad Federal de Alagoas y de la Universidad Provincial de Ciencias de La Salud de Alagoas, Brasil.

**** Graduada en Educación Física; Especialista en Educación Física Escolar, Brasil.

***** Graduado en Educación Física, Brasil. 
neutra. Rash (1989) y Hamill \& Knutzen determinan que el ángulo de inclinación del fémur tiene un valor de $125^{\circ}$, siendo denominada de coxa vara un ángulo menor de $125^{\circ}$ y de coxa valga cuando este mismo ángulo es mayor de $125^{\circ}$. Sin embargo, Werner (2000) relata que el valor de esta formación angular oscila entre $126^{\circ}$ y $128^{\circ}$. Germain (2002) nos da como valor nominal en torno a $135^{\circ}$ para el ángulo de inclinación.

Buscando verificar la influencia del ángulo de inclinación del fémur en el aparecimiento de las coxas valga y vara fueron realizadas mediciones en fémures aislados, realizando comparación de los datos obtenidos con los resultados encontrados en la literatura de forma porcentual, los valores limítrofes para clasificar la coxa como vara o valga.

\section{MATERIAL Y MÉTODO}

Cumpliendo lo que determina la Ley Federal $n^{\circ} 8.501$, de 30 de noviembre de 1992, y luego de la aprobación institucional, fueron analizados 56 fémures de cadáveres adultos, siendo 28 del lado derecho y 28 del lado izquierdo. Con un cáliper medimos el valor transverso de la cabeza (A) y del cuello del fémur (B, y determinamos el eje longitudinal de ese segmento (C). Enseguida trazamos una línea longitudinal del centro de la diáfisis femoral (D) hasta encontrar la línea del eje longitudinal del cuello, determinado el ángulo de inclinación del fémur (E), el que fue medido a través de un goniómetro (Fig. 1).

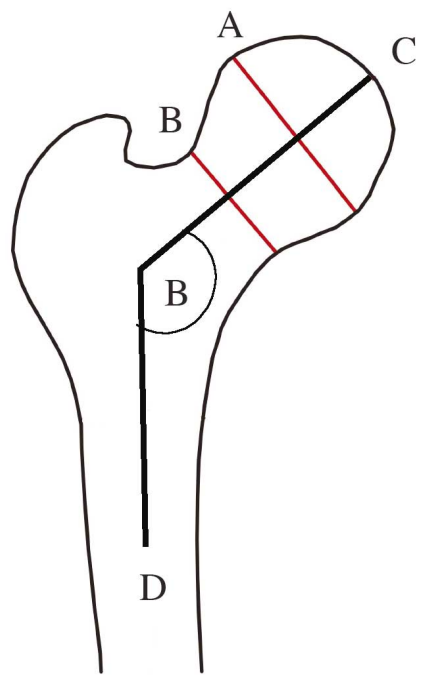

Fig. 1. Dibujo esquemático demostrando: A semirrecta de la cabeza del fémur; B - semirrecta del cuello del fémur; $\mathrm{C}$ - eje longitudinal cabeza/ cuello del fémur; D - eje longitudinal de la diáfisis femoral; E - ángulo de inclinación del fémur.
Utilizamos el patrón poblacional normal de $66 \%$ (o test Z), denominamos este intervalo como siendo aquél donde se encuentra el alineamiento ideal.

\section{RESULTADOS}

En los 28 fémures derechos encontramos que (Tabla I): en 15 fémures (54\%) el ángulo fue superior a $125^{\circ}$; en 12 fémures (42\%) la formación angular era menor de $125^{\circ}$, y en un fémur (4\%) el ángulo encontrado fue igual a $125^{\circ}$.

Tabla I. Resultados de la medición del ángulo de inclinación en 28 fémures derechos.

\begin{tabular}{lccccc}
\hline Piezas n $^{\circ}$ & Lado & Ángulo & Piezas no $^{\mathbf{0}}$ & Lado & Ángulo \\
\hline 8 & D & 112 & 30 & $\mathrm{D}$ & 130 \\
4 & $\mathrm{D}$ & 115 & 31 & $\mathrm{D}$ & 130 \\
54 & $\mathrm{D}$ & 120 & 39 & $\mathrm{D}$ & 130 \\
7 & $\mathrm{D}$ & 121 & 50 & $\mathrm{D}$ & 130 \\
14 & $\mathrm{D}$ & 121 & 55 & $\mathrm{D}$ & 130 \\
20 & $\mathrm{D}$ & 121 & 16 & $\mathrm{D}$ & 131 \\
36 & $\mathrm{D}$ & 121 & 38 & $\mathrm{D}$ & 131 \\
45 & $\mathrm{D}$ & 121 & 33 & $\mathrm{D}$ & 132 \\
47 & $\mathrm{D}$ & 121 & 34 & $\mathrm{D}$ & 132 \\
6 & $\mathrm{D}$ & 122 & 41 & $\mathrm{D}$ & 132 \\
9 & $\mathrm{D}$ & 122 & 49 & $\mathrm{D}$ & 132 \\
52 & $\mathrm{D}$ & 122 & 25 & $\mathrm{D}$ & 138 \\
51 & $\mathrm{D}$ & 125 & 53 & $\mathrm{D}$ & 139 \\
3 & $\mathrm{D}$ & 130 & 32 & $\mathrm{D}$ & 140 \\
\hline
\end{tabular}

En los 28 fémures izquierdos detectamos que (Tabla II): en 18 fémures (64\%) el ángulo presente fue superior a $125^{\circ}$, y en 10 fémur (36\%) la formación angular era menor a $125^{\circ}$.

La media de los ángulos de los 28 fémures derechos fue de $126,82^{\circ}$, y la media de los fémures del lado izquierdo de $127,53^{\circ}$, siendo la media total de $127,18^{\circ}$ (Tabla III).

En la Tabla IV demostramos estadísticamente los resultados obtenidos a partir de los 56 fémures. Medidos a través del patrón poblacional normal de 66\% (o test Z), los 56 fémures presentan valores entre $117,67^{\circ}$ y $136,67^{\circ}$. Utilizamos este intervalo como siendo el del alineamiento ideal (Fig. 2). 
Tabla II. Resultados de la medición de los ángulos del ángulo de inclinación del fémur en 28 fémures izquierdos.

\begin{tabular}{lccccc}
\hline Piezas n $^{\mathbf{0}}$ & Lado & Ángulo & Piezas n $^{\mathbf{o}}$ & Lado & Ángulo \\
\hline 10 & I & 112 & 13 & I & 131 \\
18 & I & 112 & 24 & I & 131 \\
19 & I & 120 & 29 & I & 131 \\
27 & I & 120 & 1 & I & 132 \\
2 & I & 121 & 15 & I & 132 \\
12 & I & 121 & 17 & I & 132 \\
21 & I & 121 & 28 & I & 132 \\
35 & I & 121 & 37 & I & 132 \\
22 & I & 122 & 43 & I & 132 \\
26 & I & 122 & 46 & I & 132 \\
40 & I & 128 & 44 & I & 133 \\
56 & I & 128 & 5 & I & 135 \\
48 & I & 129 & 42 & I & 138 \\
23 & I & 130 & 11 & I & 141 \\
\hline
\end{tabular}

Tabla III. Resultados de la Media para los fémures del lado derecho e izquierdo, y total de la muestra.

\begin{tabular}{lccc}
\hline Lado & $\begin{array}{c}\text { Número de } \\
\text { piezas }\end{array}$ & $\begin{array}{c}\text { Suma de los } \\
\text { ángulos }\end{array}$ & Media \\
\hline $\mathrm{D}$ & 28 & $3,551^{\circ}$ & $126,82^{\circ}$ \\
$\mathrm{I}$ & 28 & $3,571^{\circ}$ & $127,53^{\circ}$ \\
Media general & 56 & $7,122^{\circ}$ & $127,18^{\circ}$ \\
\hline
\end{tabular}

Tabla IV. Descripción estadística de las mediciones del total de la muestra.

\begin{tabular}{lcccc}
\hline $\mathrm{n}^{\mathrm{o}}$ Piezas & Media & Mediana & DS & Varianza \\
\hline 56 & 127,17 & 130 & 6,97 & 49 \\
\hline
\end{tabular}

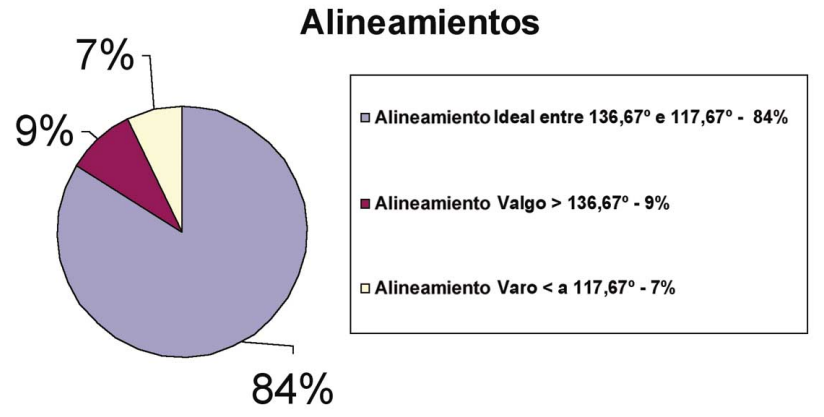

Fig. 2. Medición del alineamiento en la muestra utilizando como intervalo el alineamiento ideal.

\section{DISCUSIÓN}

Este estudio está dirigido hacia un mejor conocimiento del movimiento humano, ya que las formaciones angulares influyen, según Werner, en la "posición correcta de las piernas", pero debemos cuestionarnos con respecto a lo que es una "posición correcta", ya que, funcionalmente la marcha del Homo sapiens es bastante compleja. La literatura consultada tiene un punto en común en relación al valor de $125^{\circ}$ como siendo el ángulo de inclinación del fémur para la "posición correcta de las piernas".

Encontramos en los fémures derechos un promedio de $126,82^{\circ}$ y de $127,53^{\circ}$, para los izquierdos, siendo el promedio general de $127,17^{\circ}$. En las mediciones de los ángulos de inclinación de los 56 fémures, los valores se encontraban entre $117,67^{\circ}$ y $136,67^{\circ}$. Utilizado el patrón poblacional normal de $66 \%$ (o test Z), denominamos este intervalo, comprendido en el patrón poblacional normal, como el intervalo donde se encuentra el alineamiento ideal. En este intervalo, encontramos 47 fémures (84\%). En 5 fémures (9\%), el ángulo representaba un valor superior a $136,67^{\circ}$, y fue considerado como un alineamiento valgo. En 4 fémures (7\%) el ángulo presentaba un valor inferior a $117,67^{\circ}$, y fue considerado un alineamiento varo.

Analizando los datos reconocemos que en diferentes grados el hombre adulto puede ser portador sea de coxa vara (7\%) o de coxa valga (9\%), pudiendo aún ser portador de ambas deformaciones. Citamos como ejemplo el jugador de fútbol Manuel dos Santos, "Mané Garrincha", que es un ejemplo de portador de coxa vara y coxa valga simultáneamente. Siendo así, los profesionales de las áreas Médicas, de Educación Física y de Fisioterapia, con el conocimiento sobre estas formaciones angulares, y después de realizar análisis posturales, pueden intervenir y contribuir para la disminución, o hasta la "corrección" de las deformidades del genu varo y del genu valgo durante la infancia a través de actividades físicas dirigidas.

\section{GUSMÃO, L. C. B.; SOUSA-RODRIGUES, C. F.; MARTINS,} J. S. \& SILVA, A. J. Angle of inclination of femur in man and its relationship with coxa vara and coxa valga. Int. J. Morphol., 29(2):389-392, 2011.

SUMMARY: The authors carried out isolated mensuration in femurs to compare data with the results found in literature. The objective was to determine in percentile form, bordering values to classify the thigh as pole or valgum. Fifty six femurs of adult corpses were analyzed, determining the angle of inclination in the femur, which was measured through a Goniometry. We used the normal population standard of $66 \%$ (or tested Z): to determine in 
GUSMÃO, L. C. B.; SOUSA-RODRIGUES, C. F.; MARTINS, J. S. \& SILVA, A. J. Ángulo de inclinación del fémur en el hombre y su relación con la coxa vara y la coxa valga. Int. J. Morphol., 29(2):389-392, 2011.

which interval the ideal alignment is found. In the mensuration of inclination angles in 56 femurs., the values were between 117.67 and 136.67 degrees. Using the normal population standard of $66 \%$ (or tested Z), we verified the ideal interval (or normal) with findings between 117 and 136 degrees, the ideal alignment. In this interval we found 47 femurs $(84 \%)$. The normal determination of the angle between 117 and 135 degrees allows classifying thigh valgum, when the angle of inclination is below 117 degrees and coxa vara is above 136 degrees.

KEY WORDS: Genu valgum; Genu varum; Osteotomy of the femur; Anatomy.

\section{REFERENCIAS BIBLIOGRÁFICAS}

Field, D.; Palastanga, N. \& Soames, R. Anatomia e Movimento Humano: Estrutura e Função. São Paulo, Ed. Manole Ltda., 2000.

Germain, B. C. Anatomia para o Movimento: Introdução à Análise das Técnicas Corporais. São Paulo, Ed. Manole Ltda., 2002.

Hamill, J. \& Knutzen, K. M. Bases Biomecânicas do Movimento Humano. São Paulo, Ed. Manole Ltda., 1999.

Karpandji, A. Fisiologia Articular: Membro Inferior. Rio de Janeiro, Ed. Guanabara Koogan S/A, 2001. V. 1.

Kendal, F. P.; McCreary, E. K. \& Provance, P. G. Músculos, Provas e Funções. São Paulo, Ed. Manole Ltda., 1995.

Lapìerre, A. A Reeducação Física. São Paulo, Ed. Manole Ltda., 1982. V. II.

Rasch, P. J. Cinesiologia e Anatomia Aplicada. Rio de Janeiro, Ed. Guanabara Koogan S/A, 1989.

Rocha, P. E. C. P. Medidas e Avaliações em Ciências do Esportes. Rio de Janeiro, Ed. Sprint, 1995.

Werner, K. Atlas de Anatomia Humana: Aparelho do movimento. São Paulo, Ed. Atheneu, 2000.
Dirección para correspondencia:

Prof. Dr. Luiz Carlos Buarque de Gusmão Condomínio Aldebaran ALFA - Quadra F - N. 08

Tabuleiro dos Martins

CEP:57080-900

Maceió - Alagoas

BRASIL

Email:

Recibido : 26-10-2010

Aceptado: 18-02-2011 